MATEC Web of Conferences 40, 07004 (2016)

DOI: $10.1051 /$ matecconf/20164007004

(C) Owned by the authors, published by EDP Sciences, 2016

\title{
DSM/DMM-based Methodology for Handling Changes in Product Development Project
}

\author{
Xin Feng ${ }^{1}$, Xin Zhang ${ }^{1}$, Yan Liu ${ }^{1,2}$ \\ ${ }^{1}$ Changchun University of Science and Technology, CHINA \\ 2 The Open University, UK
}

\begin{abstract}
With the growing complexity of the business context, companies are confronting more challenging Product Development (PD) projects because of the potential risks externally brought by the partners' participation and internally accumulated through the activities' execution. In this paper, we formulate and decompose a PD project in a hierarchical way, and the obtained end elements perceived as objects are built into a number of Design Structure Matrices (DSMs) and Domain Mapping Matrices (DMMs). We also propose a conceptual model of change occurring and change propagation, base on which the potential change propagating channels are discovered through observing and tracking change occurring and change propagation in DSMs and DMMs. Associated with the depth-first and breath-first search methods executed in matrices the critical objects during change propagation are discovered and the implicit change propagating channels cross multiple fields are identified. As the exploratory results, the critical objects taking account of change propagation are highlighted as the contribution to change management in PD project.
\end{abstract}

\section{Introduction}

To introduce a final product into market through a PD project, one of the main issues is change management. Changes can be generated during the product life cycle regarding the product characteristics, the partners' collaborations or the project management. In the CoEvolution of Product and Network of Partners (CEPS) framework proposed by Zolghadri et al. in (Zolghadri, Eckert et al. 2009), the interconnection of product development and network of partners' deployment processes are highlighted by taking account of systematic influences of decisions made in product development on the supply chain design/development and vice versa. Therefore, the changes could be propagated along explicit or implicit channels linking processes to each other and across the various fields (Product design and development (Eckert et al. 2005), partner collaboration (Johnson 2009) and project management (Ibbs, Wong et al. 2001)).

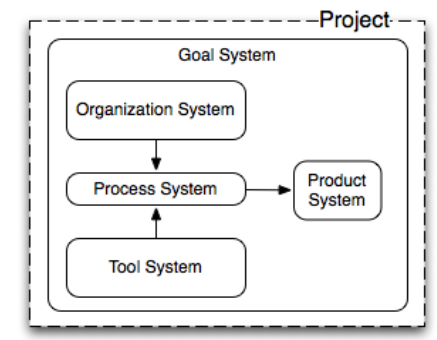

Figure 1. Five systems in PD project
Each system can be partitioned in further into subsystems formulating the specifications of their parent system, such as the structure, network, etc. With decomposing the system till obtaining the elements in the minimum granularity, the PD project can be regarded as a set of the objects concerning various viewpoints and organized in a hierarchical way. Semantically, all the subsystem obtained through dividing the five systems are called as mediate sub-systems before they are divided into the objects. Danilovic et al. in (Danilovic and Browning 2007) simplify the complexity and highlight the critical problems of the corresponding system.

With decomposing and formulating the PD project into objects, a conceptual model is then introduced based on the perceived objects to represent the change occurring and change propagation.

\section{Conceptual model}

In general understanding, we describe change as an act or process through which something becomes different. In practice, the phenomena of change are correspondingly embodied according to the specific field (i.e., product design and development, partner collaboration, project management). Changes manifest themselves in various forms and can be recognized as concerning multiple fields. Meanwhile, the fact that there exist mutual influences between changes is highlighted in our research, which can be regarded as a phenomenon that a change causes another one (Eckert, Clarkson et al. 2004). 
In order to identify changes in a qualified and quantified way, the suggested conceptual model of change occurring relies on objects from the decomposition onto the PD project. An object is an element belonging to the investigated system and is defined by a set of attributes. An object performs an action on some inputs and produces outputs. Inputs and outputs can also be characterized by a set of parameters (weight, format, color, etc.). Each parameter belongs to a predefined interval (weight between 1 and $2 \mathrm{Kg}$ for instance). The interval defines an allowable amount of variation of the parameter. Any parameter alteration within the interval is considered as normal.

Take two serial objects 1 and 2 for example. The outputs of object1 are used as inputs by object 2 . The link connecting these two objects, from the previous output to the following input, is defined as a potential channel of change propagation that could transfer the impact of a change in object1 to the object2. Considering the intersection of object1's possible outputs and object2's possible inputs, two viewpoints of observing alterations can be considered. From the object1 point of view, if due to some endogenous or exogenous alterations its generated outputs are still with the interval, no change occurs. However, from the viewpoint of object2, even though the received input is normal regarded by object1, it might be out of the interval of the input of object2. Based on the above fact, change propagation concerning the related object1 and object 2 can be described as a phenomenon that a change occurring on objectl leads to the input of object 2 out of the expected interval. The conceptual model can be illustrated in Fig. 1.2.

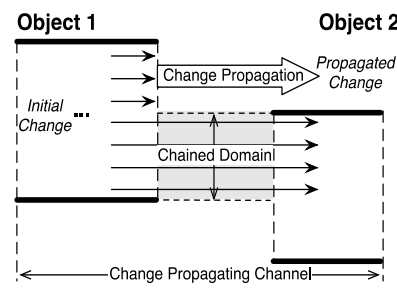

Figure 2. Conceptual model of change propagation

\section{DSM/DMM-based method}

Based on perceiving the changes and the change propagation through the conceptual model, we propose a DSM/DMM-based method to extract and represent the relationships between the objects as the potential change propagating channels.

\subsection{DSM/DMM initiation}

All the potential relationships between objects can be categorized as internal and external ones. The internal relationships refer to the ones between the objects which belong to the same parent mediate sub-system, whereas the external relationships refer to the mappings between the objects belonging to the different parent mediate subsystems (see Fig. 1.3). Correspondingly, we use DSMs to capture the internal relationships and DMMs to capture the external relationships.

In each of the DSMs/DMMs, we illustrate the relationships between the objects through qualifying the probability, which indicates how much of the probability that a change occurring in the departure object would lead to a change occurring in the destination object. The probability value is restricted between 0 (excluding 0 ) and 1 (including 1). The closer a value locates to 1 the more probable a change is to be propagated. Differing from the traditional DSMs, in this paper, the diagonal elements of the DSMs are set to zero to state that there is no change occurring in the corresponding object. As analyzing the external relationships between the objects belonging to the different parent mediate sub-systems, the DMMs are also initiated through filling the probability values restricted as in DSMs.

In the filled DSMs and DMMs, the treatments, such as sequencing and clustering (Browning 2001), are operated to eliminate the potential conflicts in project scheduling, activity sequencing, system architecting or engineering such as inter-dependency, iterations, etc.

Given that the initiated DSMs extract the relationships between objects, one of the diagonal elements filled with zeros is modified into a candidate value from a given set, i.e., 1, 2, and 3 to illustrate change occurring, in which the values demonstrate the impact brought by the change to the given object. The values demonstrating change occurring are named as change qualification value. The higher the change qualification value is, the more impact is brought into the concerned object.

\subsection{Change propagation search}

Through modeling the PD project in a hierarchical way, we obtain a direct acyclic graph, i.e., a tree taking the global PD project as the root, all the mediate systems as the branch and all the objects as the leaves. Fig. 1.3 displays an example of the hierarchical model as well as initiations of DSMs and DMMs.

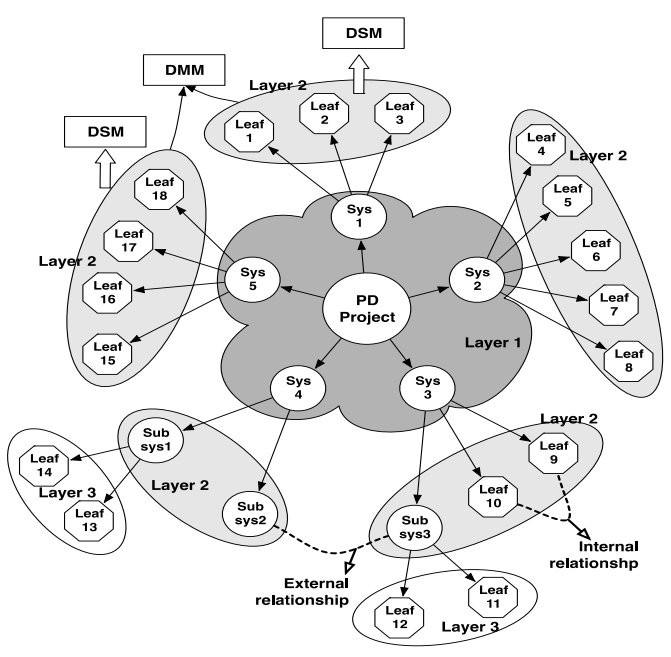

Figure 3. Hierarchical Model of PD Project 
As collecting the objects belonging to the same parent mediate system to build DSMs and the objects belonging to the different parent mediate systems to build DMMs, the probability values of transmitting change propagation from one object to another one are filled in DSMs and DSMs. Then an algorithm is executed to obtain the critical object(s) and the mediate system(s) to identify and discover the critical activities, components, resources, etc. in further to improve the change management of PD project.

The algorithm is proposed as:

1. The algorithm is invoked when there is a change occurring in an object, which is illustrated by modifying the zero value into the corresponding change qualification in the diagonal cell of the concerned DSM. The object where the change occurs is highlighted and a queue with recording the object as the head is created;

2. Within the concerned DSM, a breadth-first search departed from the highlight-ed object is executed to search the direct related object(s), during which the change qualification value of change occurring is multiplied with the probabil-ity value(s) locating in the corresponding cell(s);

- If only one related object from the search is found, then this object is in-serted into the queue as the tail (initiated in step 1);

- If more than one related objects from are found, then the existing queue is duplicated as the ones taking the found objects as the tails respectively.

The change qualification value brought by the departure object is transferred and multiplied with the probability value in the current cell, and the result value (named as qualification value of change propagation) is also recorded in the queue;

3. The breath-first-like search is kept executing within the DSM until either of the following conditions are satisfied:

- If the latest found object has appeared in the current queue, then termi-nate adding it at the end of the queue;

- If no new object is found through the search, then terminate searching.

4. Through the search within the given DSM, one or more queues are generated with the concerned objects as the nodes and the links illustrated with the quali-fication values of change propagation between each two of objects. Then a threshold is used to compare with all the values in the queues, which can be ob-tained from interviewing with the field experts. If one qualification value of change propagation is lower than the threshold, then it implies the change caused is within acceptable impact or low occurring probability.

- From the head of each of queues, if the threshold is larger than the qualifi-cation value of change propagation between the given two nodes (i.e., from departure object to destination object), then the rest of the queue including the destination node is removed;

- If the threshold is smaller than any of the qualification values of change propagation along the queue, then the queue is left as before.

5. After filtering the queue(s) of the given DSM, the tail(s) of all the queues are regarded as the departure object(s). If there are more than one generated queues with the same object as the tails, then only the maximum qualification value of change propagation is selected to be transformed for the following op-erations;

6.If there exists at least one DMM taking the given DSMs as the candidate-mapping domains, then a depthfirst search is executed within the concerned DMM.

7. With the depth-first search, the object(s) belonging to other domain can be found. Along the search, the qualification values of change propagation are computed and recorded as being operated in the previous procedure (illustrated in step 3);

8. As result, the object(s) found through the search in DMM is (are) selected the departure node(s) and processed in the mapped DSM(s) through repeating from step 1 to step 7.

Through the treatments in the matrices according to the above algorithm, we achieve the following exploratory results:

- Each of the generated queues belonging to a DSM represents one of the poten-tial channels of change propagation.

- Taking account of all the generated queues belonging to a DSM, the frequency of all the objects appearances through the search composes a rank of criticali-ties, in which the object with higher frequency should be highlighted in the high priority during change management in a PD project Because during the practical process, the highlighted objects are more probable influenced by change occurring, and meanwhile they should be given priority to be managed.

- In further considering multiple DSMs mapped through $\operatorname{DMM}(\mathrm{s})$, besides rank-ing the criticalities of the objects, the potential channel(s) compose(s) a global network as the suggestion to cope with change and change propagation in a PD project. Furthermore, the implicit influence transferred between various do-mains can be identified thanks to the global potential channels.

\section{Conclusion}

In this paper, we formulated and decomposed a PD project in a hierarchical model and proposed a conceptual model of change occurring and change propagation based on the end elements (i.e., objects) obtained from the decomposition. With the objects, DSMs and DMMs were initiated to start our observation of change propagation in the PD project. Associated with the depth-first and breath-first search methods, the critical objects were discovered and the implicit change propagating channels cross multiple fields were identified.

\section{Acknowledgement}

This research was performed partly by the support of International Joint Research Project funded by Jilin Provincial Science and Technology Department: Research on process of Upgrade-to-Performance of complex systems with long service duration. 


\section{References}

1. T. R. Browning, E. Fricke, H. Negele "Key concepts in modeling product develop-ment processes" Systems Engineering 9(2): 104-128 (2006)

2. M. Danilovic, T. Browning "Managing complex product development projects with design structure matrices and domain mapping matrices" International Journal of Project Management 25(3): 300-314 (2007)

3. C. Eckert, P. J. Clarkson, W. Zanker, "Change and customisation in complex engineering domains." Research in Engineering Design 15(1): 1-21 (2004)

4. C. Eckert, P. J. Clarkson, C. Earl, "Predictability of Change in Engineering: A Com-plexity View". ASME 2005 International Design Engineering Technical Conferences \& Computers and Information in Engineering Conference, Long Beach, California, USA, ASME (2005)

5. EIA, "EIA STANDARD: Processes for Engineering a System". Wilson Boulevard Arlington, VA 22201, EIA (1999)

6. C. William. Ibbs, K. Wong. Clarence, Young Hoon Kwak, "Project change management sys-tem". Journal of Management in Engineering 17(3): 159$165(2001)$

7. P. F. Johnson, M. R. Leenders, "Changes in supply leadership." Journal of Pur-chasing and Supply Management 15(1): 51-62 (2009)

8. T. U. Pimmler, S. D. Eppinger, "Integration Analysis of Product Decompositions". 6th International Conference on Design Theory and Methodology, Minneapolis, MN (1994)

9. M. Zolghadri, C. Eckert, S. Zouggar, P. Girard, "A Taxonomy of Collaboration in Supply Chains". International Conference on Engineering Design ICED'09. Stanford University, Stanford, CA, USA (2009) 